\section{Organización social del cuidado en Bolivia y Chile: Estado y cuidadanía*}

Social care in Bolivia and Chile: Government and cuidadanía

\section{SANDRA LEIVA-GÓMEZ}

\section{Resumen}

El presente artículo examina la organización social del cuidado en Bolivia y Chile. El análisis cobra relevancia en el contexto de las cadenas globales de cuidado entre ambos países, las que surgen como una estrategia ante la crisis de cuidado que crecientemente afecta a la sociedad chilena. A través del enfoque de la cuidadanía se examina la participación estatal

\footnotetext{
Esta contribución se inscribe en el proyecto ANILLOS CONICYT SOC 1109 "Relaciones transfronterizas entre Bolivia y Chile: Paradiplomacia y prácticas sociales 1904-2004", desarrollado por el Instituto de Estudios Internacionales, INTE, Universidad Arturo Prat, en conjunto con el Instituto de Estudios Avanzados, IDEA, Universidad de Chile. El presente trabajo constituye una versión ampliada de la ponencia presentada al XIII Encuentro Bolivia Chile, en La Paz, UMSA, 9 al 11 de Julio de 2014.

** Instituto de Estudios Internacionales, Universidad Arturo Prat, Iquique, Chile. Correo electrónico: sandleiva@gmail.com
}

como una arista de la organización social del cuidado, observando específicamente la provisión estatal de cuidado infantil en estos países. Los resultados indican que tanto Bolivia como Chile han realizado avances en esta materia, plasmados en programas públicos, leyes y declaraciones programáticas. Ambos Estados están, así, dando pasos hacia la instauración de un modelo de cuidadanía, en el que la corresponsabilidad social comienza tímidamente a establecerse. No obstante, aún persisten insuficiencias de cobertura de los programas y una falta de concordancia entre las prioridades enunciadas y las leyes que deberían sustentarlas.

Palabras clave: organización social del cuidado, cadenas globales de cuidado, Chile, Bolivia, migración, cuidadanía.

\section{Abstract}

This article examines the organization of social care in Chile and Bolivia. The analysis takes on special relevance in the context of global care chains that exist between the two countries, which emerge as a strategy for managing the crisis of care that is increasingly affecting Chilean society. Through the lens of cuidadanía, the author examines government participation as an element of social care. Specifically, the article analyzes the provision of public childcare in the two nations. The results indicate that both Bolivia and Chile have made progress in this area, as evidenced by public programs, laws, and program statements. Both governments are taking steps towards the installation of a model of cuidadanía in which social co-responsibility timidly begins to be established. However, the coverage of the programs is not complete and there is a lack of consistency between the 
priorities that have been identified and the laws that must be implemented in order to support these efforts.

Key words: social care, global care chains, Chile, Bolivia, migration, cuidadanía.

\section{Introducción}

A partir de los 90, varios países de América Latina y el Caribe se constituyen en países de origen y/o destino de una modalidad de migración protagonizada por mujeres, quienes se dirigen hacia países de la misma región o de Europa para insertarse en el servicio doméstico, realizando labores fundamentalmente de cuidados (Anderson 2012; Molano, Robert y García 2012; Carcedo, Lexartza y Chaves 2011; Sanchís y Rodríguez 2011). El aumento de la participación laboral femenina ha inducido a las mujeres a buscar diversas estrategias para conciliar su vida familiar y laboral.

Al restarse normalmente los varones de las tareas de cuidado ${ }^{1}$, y al no existir suficientes políticas públicas que proporcionen tales servicios, éstos quedan por lo general en manos de las mujeres. Así, como estrategias de conciliación, algunas buscan trabajos flexibles, informales o de medio tiempo, otras dejan a sus niños al cuidado de su abuela, en tanto que otras contratan personal de servicio doméstico en el mercado, el que puede ser desempeñado por una persona nacional o extranjera. Normalmente, las mujeres extranjeras que trabajan en un hogar desempeñando labores de cuidado, tienen a su vez hijos pequeños en

Los estudios de uso del tiempo muestran que los varones se dedican escasamente a las tareas de cuidado (Aguirre, García y Carrasco 2005). el país de origen, los que quedan a cargo de otra persona, conformándose de esta manera cadenas globales de cuidado.

Aun cuando entre Chile y Bolivia no existe una investigación que analice las cadenas globales de cuidado entre ambos países ${ }^{2}$, el fenómeno cobra especial relevancia si se considera el aumento de la migración boliviana en el país, que de 24.126 residentes en el año 2009 (Departamento de Extranjería y Migración 2010) pasó a 34.201 en 2014 (Fundación Scalabrini 2014). A lo anterior se suman los estudios que indican que las mujeres bolivianas se insertan en una alta proporción en el servicio doméstico (Gavilán y Tapia 2006; Carrasco 1998).

En este artículo se analiza un aspecto específico de las cadenas globales de cuidado: la organización social del cuidado en Bolivia y Chile a la luz de las reflexiones teóricas proporcionadas por el enfoque de la cuidadanía (Bodelón 2010; Rodríguez 2010). La organización social del cuidado (Daly y Lewis 2000; Lewis 1998) considera la prestación de cuidados que realizan las diversas instituciones sociales. De esta manera, se considera el aporte del Estado, el mercado, las familias y la comunidad al bienestar global en la atención a personas dependientes, así como las interrelaciones entre ellos.

En las investigaciones sobre las cadenas globales de cuidado se ha estudiado la organización social del cuidado tanto en los

\footnotetext{
Los estudios sobre el tema en Chile fundamentalmente han abordado las cadenas entre Chile y Perú (Acosta 2013a, Acosta 2013b; Arriagada y Todaro 2012; Arriagada y Moreno 2011; Setién y Acosta 2011), mientras que las investigaciones en Bolivia se refieren fundamentalmente a las cadenas entre Bolivia y España (Guaygua 2010; Hinojosa 2010, 2009; Salazar, Jiménez y Wanderley 2010; Tapia 2010a, Tapia 2010b).
} 
países que expulsan como en los que atraen a las mujeres migrantes (Acosta 2015; Pérez y López 2011). El objetivo de este trabajo es, por tanto, analizar la organización social del cuidado en las prestaciones estatales en Bolivia como país de origen y Chile como país de destino, en lo que se refiere específicamente a la provisión de cuidado infantil.

Al estudiar la participación estatal en la prestación de cuidado, normalmente suelen emplearse los enfoques del Estado de Bienestar (PfauEffinger 2014; Martínez Franzoni y Voorend 2013), o el enfoque de derechos (Pautassi 2013; Montaño etal.2012). La utilización del enfoquede la cuidadanía para analizar la organización social del cuidado constituye, por ende, una novedad teórica que este artículo aspira a presentar. Esta perspectiva da cuenta de un modelo de ciudadanía que reconoce la interdependencia de las personas y su naturaleza interrelacional, lo que se ve reflejado en las necesidades de cuidado que todos los seres humanos tienen en algún momento de sus vidas. Según la perspectiva de la cuidadanía (Rodríguez 2010), el Estado debe apoyar las relaciones que favorecen nuestra autonomía, y debe, asimismo, propender a impedir las relaciones de poder que entorpezcan tal autonomía, la que posibilita la capacidad de las personas de autonormarse y formar su identidad.

El artículo se estructura de la siguiente manera: En la primera sección se explica el concepto de cuidado y su legitimación en las ciencias sociales. Posteriormente, se analiza la crisis del cuidado y el papel que en ella desempeñan las cadenas globales de cuidado, conformadas para sostener cotidianamente la vida tanto en hogares de origen como de destino. A continuación se tematiza la crisis de cuidado en
Chile y la contratación de mano de obra femenina boliviana. Luego se analiza la organización social del cuidado como concepto que permite analizar las prestaciones entregadas por diversos actores para la realización de labores de cuidado. Enseguida se presenta un apartado teórico que analiza la responsabilidad del Estado en la implementación de políticas públicas para proporcionar servicios de cuidado a la población, revisando para ello el enfoque de la cuidadanía, que subraya una ciudadanía civil teniendo en consideración la interdependencia de los seres humanos. El texto continúa analizando la organización social del cuidado en Bolivia y en Chile desde la perspectiva de la cuidadanía, para finalizar con las conclusiones.

\section{El trabajo de cuidados}

El término "cuidado" es relativamente nuevo en las disciplinas sociales. Los primeros estudios en sociología que se refieren al tema de cuidados los encontramos en la década del 80, los que utilizaban los términos de "el cuidado", "los cuidados" o "el trabajo de cuidados", aunque ninguna de estas expresiones es una fiel traducción del inglés "care" (Carrasco, Borderías y Torns 2011). El desarrollo del concepto está relacionado con la ruptura epistemológica del concepto de trabajo que se dio en las Ciencias Sociales a fines de la década de los 80. A raíz de los debates europeos, se incluyó el trabajo doméstico en la categoría de trabajo. Esto supuso reconocer que existe un concepto de trabajo más amplio que aquél que lo reduce a la actividad laboral o empleo, teniendo en consideración el trabajo doméstico o reproductivo que las dueñas de casa realizan al interior de sus hogares (Torns 2008), el 
que había sido largamente naturalizado e invisibilizado.

El estudio de los cuidados en la sociología se consolidó cuando fue presentado como un elemento clave de las políticas sociales. Esa nueva visión aparece a partir de los 90 en la intervención sociopolítica, donde se incluyen las especialistas europeas en las políticas de los Estados de Bienestar (Carrasco, Borderías y Torns 2011). Las académicas británicas han ideado el concepto de 'social care' para que el Estado de Bienestar considere cómo se organiza socialmente el cuidado. El término 'social care' ha sido traducido entonces como organización social del cuidado (Carrasco, Borderías y Torns 2011; Torns 2008).

El cuidado implica una interacción entre las personas, y aunque incluye también aspectos de lo que se puede llamar cuidado material, como lavar, trasladar, alimentar, vestir, etc., se caracteriza por incluir tareas de formación y socialización (Acosta 2011). El cuidado está basado en una relación, y no es solamente una obligación establecida por la ley, pues incluye emociones que se expresan en las relaciones familiares, contribuyendo a la vez a construir y mantener esas relaciones (Aguirre 2007). Según Batthyáni (2004), el trabajo de cuidados se compone de cuidado material, que es la realización del trabajo, de cuidado económico, que dice relación con el costo, y de cuidado psicológico, en tanto se da un vínculo afectivo con la persona cuidada.

El trabajo de cuidados se inserta dentro del trabajo no remunerado de las mujeres, pero ello no quiere decir que este trabajo no tenga costos. Lo que sucede es que los costos están ocultos y generalmente no se contabilizan. El trabajo de cuidados realizado por los hogares es considerado como si fuera una oferta infinita: ello implica trabajo gratuito de las mujeres (Carrasco, Borderías y Torns 2011). En efecto, desde hace ya más de una década, se ha reconocido el aporte que las mujeres realizan a las economías de los países (Esping-Andersen 1999), contribuyendo de esta forma a generar bienestar.

Los cambios en las expectativas de las mujeres, la incorporación de éstas al mercado laboral, el mandato de género que les entrega la responsabilidad de realizar tanto las labores del hogar como el cuidado de personas dependientes, entre otros factores, las llevaron a desarrollar diversas estrategias de conciliación entre la vida familiar y laboral (Durán 2004), entre ellas la reducción de la jornada de trabajo, la ayuda de mujeres familiares -como la madre o la hermana-, o la contratación de personal de servicio doméstico. Si bien es cierto este servicio había sido desempeñado tradicionalmente en Chile por personas de la misma nacionalidad, en los últimos años está siendo proporcionado por extranjeras de origen peruano (Stefoni 2009), apareciendo, de acuerdo a Salazar (2001), la migración femenina como respuesta a la crisis de los cuidados.

\section{Crisis de cuidado y cadenas globales de cuidado}

La crisis de cuidado se experimenta en sociedades en que, fundamentalmente, la mujer ha incrementado su participación en el mercado laboral, disminuyendo, por tanto, el tiempo que antes tenía disponible para el cuidado de las personas. Esta "care drain" (Bettio, Simonnazzi y Villa 2006), o 
en castellano "fuga de cuidado", debe ser cubierta por otras personas, o bien, por alguna institución privada o estatal. De manera más amplia, la crisis de cuidado es un complejo proceso de reorganización de los trabajos de cuidados, el que descansa según el mandato de género mayoritariamente en las mujeres. Sin embargo, las mujeres cada vez más carecen de la capacidad de responder a las necesidades de cuidados de las personas (Pérez 2006). La crisis de cuidados en los países del norte ha originado la migración de mujeres provenientes de los países del sur hacia los países más desarrollados, ofreciendo su trabajo como "cuidadoras". Este proceso fue llamado por Hochschild (2001) "cadenas mundiales de afecto y asistencia", el que ha sido traducido como "cadenas globales de cuidado" (Carrasco, Borderías y Torns 2011).

Las cadenas globales de cuidado constituyen hoy uno de los fenómenos más paradigmáticos del actual proceso de feminización de las migraciones (Pérez 2007). El concepto refiere la cadena en que la mujer autóctona es sustituida en el cuidado de sus hijos y dependientes por la mujer migrante, la cual es sustituida a su vez en el cuidado de sus hijos por otra mujer (sea la abuela, la hermana, la suegra) (Acosta 2011). Se trata de cadenas transnacionales, que se conforman para sostener cotidianamente la vida. En estas cadenas los hogares se transfieren trabajos de cuidados de unos a otros, basados en ejes de poder asociados con el género, la etnia, la clase social, así como el lugar de procedencia (Pérez 2009).

El surgimiento de las cadenas globales de cuidado se explica por la confluencia de dos crisis: la crisis de cuidados, referente a un déficit en las tareas de cuidado, la cual se da en el país de destino, y crisis económica, que alude a la insuficiencia de ingresos para mantener a la propia familia, la cual tiene lugar en el país de origen (Arriagada y Todaro 2012). Ello provoca que en las cadenas, las mujeres que vienen a satisfacer las demandas de cuidado provengan por lo general de países menos desarrollados. Esta transnacionalización del trabajo de cuidados agudiza las desigualdades entre las mujeres, en lo que se refiere fundamentalmente a cuestiones de clase, etnia o raza. Se produce, entonces, una suerte de triple discriminación que afecta a las mujeres que provienen de otros lugares: mujer, inmigrante y trabajadora (Parella 2003).

La migración femenina desencadena importantes transformaciones en los hogares: en primer lugar, se produce una redistribución del trabajo de cuidados de los familiares que permanecen en el país de origen; en segundo lugar, sedesarrollan los hogarestransnacionales. Surge de esta manera un nuevo modelo de familia: la familia transnacional, en la que a pesar de que sus miembros viven separados a través de fronteras nacionales, mantienen frecuentes relaciones entre sí. La transnacionalidad se entiende como un fenómeno en que tanto la vida como los proyectos de los migrantes no se ubican en un espacio único, sino que se forjan y mantienen relaciones simultáneas, uniendo sus sociedades de origen con las de destino (Acosta 2011). La familia transnacional da lugar a prácticas de cuidado transnacional, constituyéndose la maternidad a distancia en una de las prácticas más estudiadas. Tal maternidad transnacional implica una construcción distinta del significado de la maternidad para las madres migrantes (Stefoni 2013; Ariza 2012; Hondagneu-Sotelo y Ávila 1997). 


\section{Crisis del cuidado en Chile y migración boliviana}

En Chile se está produciendo una crisis de cuidado, la que se explica por diversos factores. Entre ellos se cuenta, en primer lugar, una mayor incorporación de las mujeres al mercado laboral, lo que se refleja en el constante crecimiento de la población económicamente activa femenina: de un $32,5 \%$ en 1990 , asciende a un $43,5 \%$ en el año 2011 (MIDEPLAN 2012). En segundo lugar, asistimos a un cambio en la estructura familiar, a partir del cual el hogar nuclear biparental está perdiendo peso, y lo está ganando la familia monoparental, donde mayoritariamente hace de cabeza una jefa de hogar. En tercer lugar, se observa un envejecimiento de la población, dado por la disminución de la tasa de natalidad y por el aumento de la esperanza de vida (Acosta 2011; Arriagada y Moreno 2011). Al respecto, deben considerarse sobre todo aspectos culturales, como la división sexual del trabajo imperante en la sociedad que asocia el trabajo productivo al hombre y el reproductivo a la mujer (Batthyány, Genta y Perrotta 2013; Torns 2008), determinando que el mandato de género asociado a estos roles sea un factor clave en la crisis de cuidado observada.

La menor oferta de cuidadoras aparejada a la creciente demanda de cuidado, al no ser satisfecha por la familia, el mercado o el Estado, está siendo proporcionada por mujeres inmigrantes en Chile. Estamos asistiendo a una feminización de las migraciones, que oscila entre el $50 \%$ y el $60 \%$ en migrantes provenientes de Perú, Bolivia y Argentina, conformándose lo que se ha dado en llamar una migración sursur. En cuanto a la motivación para migrar de las mujeres a nivel intrarregional, hay que considerar además del trabajo disponible, la cercanía geográfica y el menor costo que representan los viajes (Acosta 2011). En mujeres inmigrantes peruanas, el trabajo doméstico es la principal actividad económica desarrollada, lo que puede observarse en el censo del año 2002 , según el cual el $70 \%$ de las mujeres peruanas inmigrantes se emplea en el servicio doméstico (Martínez 2003).

Se conocen en Chile dos investigaciones sobre cadenas globales de cuidado: el estudio desarrollado por Arriagada y Todaro (2012), y el estudio llevado a cabo por Acosta (2013a, 2013b, 2011), los que analizan las migraciones de mujeres peruanas que se insertan en Santiago para proporcionar servicios de cuidado. Sin embargo, no existen investigaciones que analicen la prestación de servicios de cuidado de inmigrantes bolivianas en Chile, aun cuando comienza a haber evidencia de que este fenómeno se está presentando en el país. La tendencia a la feminización de la migración boliviana observada en los últimos censos (Tapia 2012), junto con los estudios que indican la inserción de las migrantes bolivianas en el servicio doméstico en lquique (Arriaza 2008; Gavilán y Tapia 2006) y en Arica (Carrasco 1998; González 1998), considerando además la alta tasa de fecundidad boliviana, dan sustento a la conformación de cadenas globales de cuidado protagonizadas por mujeres bolivianas en Chile.

Chile comparte más de 800 kilómetros de frontera con Bolivia, siendo los departamentos colindantes La Paz, Oruro y Potosí. La migración fronteriza se ve facilitada por las normativas legales vigentes, dentro de las cuales cobra importancia el Acuerdo de Residencia de los Nacionales de los Estados Partes del MERCOSUR, vigente desde el año 2009. Según este acuerdo, los habitantes de Argentina, 
Bolivia, Brasil, Paraguay y Uruguay pueden obtener una visa de residencia temporaria por un año, sin que deban contar con un contrato de trabajo cuando realizan la solicitud (Tapia y Ramos 2013).

Diversas fuentes avalan el aumento de la migración boliviana a Chile en los últimos años. De acuerdo a las proyecciones entregadas recientemente por el Departamento de Extranjería y Migración, se contabilizan 34.201 bolivianos en el país que poseen una residencia de larga duración, correspondiendo a un 7,7\% del total de extranjeros (Fundación Scalabrini 2014). La mayor afluencia de población boliviana a Chile es confirmada por el Consulado General de Chile. A través de la aplicación de un formulario sobre el número de bolivianos en Chile, el Consulado estimó que habitan aproximadamente 50.000 bolivianos con residencia estable en Chile, de los cuales unos 20.000 cuentan con permisos de residencia y cerca de 30.000 se encontrarían en condiciones irregulares (Organización Internacional para las Migraciones 2011). Además, según los datos entregados por el último Censo realizado en Bolivia, Chile constituye el cuarto país de preferencia para migrar de los ciudadanos bolivianos, contabilizándose 31.313 personas, lo que corresponde a $5,6 \%$ de los emigrantes (Instituto Nacional de Estadísticas 2014).

Los extranjeros de origen fronterizo se insertan en distintos tipos de trabajo en Chile de acuerdo a su sexo. Mientras los hombres se dedican a la construcción y a la agricultura, las mujeres migrantes se emplean generalmente en el servicio doméstico (Gavilán y Tapia 2006; González 1998). Los varones realizan trabajos riesgosos y pesados, y las mujeres trabajos socialmente devaluados (Tapia 2012).
El trabajo en el interior de los hogares es un ámbito de difícil control, por lo que se tiene poca información sobre las condiciones de trabajo de los migrantes fronterizos en esta área (Berganza y Cerna 2011). Los trabajadores peruanos y bolivianos más calificados que ingresan a Chile por el norte se desplazan hacia el centro, permaneciendo en esa zona los menos calificados. Ello implica que las principales fuentes de trabajo de las inmigrantes sea el servicio doméstico (Vergara 2005).

Según un estudio llevado a cabo por Arriaza (2008), en la Pastoral Migratoria de lquique se contabilizaban 483 bolivianos, correspondiendo la inmensa mayoría a mujeres. El 95\% de las inmigrantes se inserta en el trabajo doméstico. Un estudio más reciente acerca de los migrantes registrados en la Pastoral Migratoria de lquique lo constituye el de Ramos y Urbina (2012), en el que se observa la misma tendencia hacia la preferencia de las migrantes por el trabajo doméstico. Al no existir estudios sobre las condiciones de llegada de mujeres migrantes bolivianas, la Pastoral de Migraciones de lquique, INCAMI, presenta una interesante opción de conocimiento. Se trata de una institución que acoge a un promedio de 1.000 mujeres anualmente. El estudio de Ramos y Urbina (2012) realiza una revisión de los registros de ingreso de los migrantes en el periodo de enero a junio del año 2010. Se registran 446 mujeres inmigrantes ingresadas a la pastoral en ese periodo. De ellas, 374 son bolivianas, lo que corresponde a un $75 \%$. Un $98 \%$ de las migrantes registradas en el periodo indicado manifiesta una preferencia por trabajar en el servicio doméstico.

Los cuidados son necesarios, la sociedad necesita que alguien se haga cargo del cuidado 
de niños, adultos mayores, discapacitados y personas enfermas. Es válido, por tanto, preguntarse qué respuesta entrega la sociedad a esta necesidad y, fundamentalmente, cómo el Estado se hace partícipe en ella. A continuación se examina el concepto de organización social del cuidado, en tanto da cuenta de la distribución de la carga global de cuidados en la sociedad.

\section{Organización social del cuidado}

Fueron académicas británicas estudiosas del Estado de Bienestar quienes idearon el concepto de 'social care', traducido como organización social del cuidado, reclamando que el Estado dictara políticas para llevar a cabo las tareas de atención (Carrasco, Borderías y Torns 2011; Daly y Lewis 2000; Lewis 1998). Con el término 'social care' se referían a los arreglos que el Estado de Bienestar debe realizar a fin de proporcionar cuidado a las personas dependientes en una sociedad, sean niños, ancianos o discapacitados. No solamente se necesita una redefinición del cuidado y de las políticas orientadas a su realización, sino que es preciso redefinir el vínculo fundador del propio Estado de Bienestar, visión que aparece en la intervención sociopolítica en la década de los 90 (Carrasco, Borderías y Torns 2011).

El término 'social care' fue desarrollado por Lewis (1998) y por Daly y Lewis (2000), quienes entienden que se trata de un concepto tridimensional. En efecto, se reconoce una vertiente de cuidados como trabajo, otra como un marco normativo de obligación y responsabilidad, y una tercera como actividad que involucra costos financieros y emocionales, atravesando tanto la esfera pública como la privada. Así considerado, las autoras definen el 'social care' como las actividades y las relaciones que hacen posible la satisfacción de las necesidades tanto físicas como emocionales de las personas adultas dependientes y de niños y niñas, considerando los marcos normativos, económicos y sociales en que tales actividades se desarrollan (Daly y Lewis 2000).

La organización social del cuidado puede ser estudiada tanto en términos macro como micro. A un nivel macro se analiza la prestación de cuidado otorgada por los diferentes sectores de la sociedad -el Estado, el mercado, las familias y la comunidad-, así como la interrelación entre estos actores. De este modo, la división del trabajo, la responsabilidad y los costos asociados constituyen el punto de partida analítico fundamental en este nivel. Lo que interesa es analizar la contribución de cada sector a la carga global de cuidados que la sociedad requiere. A nivel micro se investigan los procesos de las personas que prestan y reciben cuidado, estableciendo el tipo de relaciones existentes entre la persona cuidadora y la receptora de cuidado, las condiciones económicas y sociales bajo las cuales se desarrollan las labores de cuidado, así como el impacto para las personas que reciben las prestaciones de cuidado (Daly y Lewis 2000; Lewis 1998).

Al aplicar la organización social del cuidado al estudio de las cadenas globales de cuidado se incorporan ambos eslabones (Gonzálvez 2013; Molano, Robert y García 2012; Pérez y López 2011). De esta manera, a nivel macro se investigan las instituciones de los países de origen y de destino que conforman la infraestructura de cuidados, mientras que a nivel micro se investigan los procesos de las personas que prestan y que reciben cuidado tanto en los hogares 
de origen como de destino. En este artículo se aborda el aspecto macro, proporcionando antecedentes para comprender la organización social del cuidado en Bolivia como país de origen y en Chile como país de destino.

El análisis de la organización social del cuidado comprende tanto la demanda como la oferta del cuidado, vale decir, quiénes necesitan servicios de cuidado -niños, adultos mayores, personas con discapacidad o enfermas-, así como las instituciones que entregan servicios de cuidado, comprendiendo el Estado, el mercado, las familias y la comunidad organizada. En este trabajo se restringe el análisis a la oferta de cuidado por parte del Estado, tanto en lo relativo a programas que ofrecen atenciones, como las leyes que facilitan las labores de cuidado. El examen de las prestaciones estatales se focaliza exclusivamente en el cuidado de niños. En el apartado siguiente se entregan elementos para comprender un enfoque teórico que le otorga un rol preponderante al Estado en materia de cuidado: la cuidadanía, y posteriormente se analiza el papel del Estado en la organización social del cuidado de niños en Bolivia y en Chile a partir de las aportaciones teóricas de la cuidadanía.

\section{Enfoque teórico: Cuidadanía como nuevo modelo de ciudadanía}

El cuidado puede ser comprendido teóricamente desde diversos enfoques. Tal vez los más conocidos sean el enfoque de derechos y el de los Estados de Bienestar. El primero, promovido en la última década fuertemente por la CEPAL (Montaño et al. 2012; Montaño 2010; Pautassi 2010), postula que el cuidado es un derecho que todas las personas dependientes tienen, ya se trate de infantes, adultos mayores, enfermos o personas con discapacidad. El Estado debe, por tanto, garantizar su cumplimiento, promoviendo políticas públicas adecuadas y estableciendo arreglos institucionales tendientes a este fin.

Por su parte, desde el enfoque de los Estados de Bienestar, el cuidado plantea la necesidad de desarrollar un modelo para comprender las dinámicas sociales asociadas a dichos Estados que no sea neutro al género, sino que lo incluya como una dimensión explicativa. Así, el genderfare combina el análisis de los Estados de Bienestar (welfare) con la perspectiva de género (gender) (Duncan y Pfau-Effinger 2000), reivindicando la presencia de las mujeres en la comprensión global del bienestar. El enfoque reconoce las labores que las mujeres desarrollan cotidianamente para mantener el bienestar al interior de sus familias, contribuyendo así a las economías de los Estados de Bienestar (Esping-Andersen 2002).

En esta dirección, un enfoque menos conocido para la comprensión teórica del cuidado es el de cuidadanía, el cual reconoce las necesidades de relación y de cuidado de los seres humanos en el ejercicio de su ciudadanía (Bodelón 2010), teniendo en cuenta la interdependencia de los individuos (Rodríguez 2010), en vez de partir de la ficción de que las personas son independientes, como hasta el momento se había hecho. En este artículo se aborda el cuidado utilizando este último enfoque teórico.

El concepto de cuidadanía nace como fruto de un error en España en el año 2004, en donde un centro vecinal en Sevilla, en vez de escribir ciudadanía, anotó cuidadanía (Rodríguez 2010). Posteriormente, los movimientos sindicales comienzan a prestarle atención a este concepto 
desde una dimensión de género (Rodríguez 2010). En efecto, en 2005 la Confederación General del Trabajo realizó una defensa del término definiendo un nuevo modelo ciudadano. Según un manifiesto emitido por ésta, la cuidadanía se entiende como una forma de reivindicar a los sujetos en una sociedad que sitúe a la vida en el centro, que se organice de acuerdo a las necesidades de las personas, sin excluir a nadie. Es necesario reconocer a la gente en su diversidad, en su pluralidad, sabiendo que no puede ser reducida a normas. Desde ese reconocimiento será posible destronar a los mercados, transformar la organización social, centrarse en las necesidades de las personas, en los cuidados de las personas como un nuevo eje. Toda persona debe ser, o poder ser, parte de una red horizontal de cuidados. La cuidadanía es, pues, universal (Pérez 2006).

El enfoque de cuidadanía constituye una crítica al modelo de ciudadanía marshalliano existente, el que se presenta como irreal pues parte de supuestos falsos. La comprensión de la ciudadanía de Marshall (1963), que considera la ciudadanía civil, política y social, es un modelo construido basándose en un hombre trabajador. Es, por lo tanto, un modelo con un sesgo de género masculino (Rodríguez 2010). Pateman (1988) acuñó la expresión "contrato sexual", como condición de posibilidad del pacto social que había sido forjado en el marco de una sociedad libre, igualitaria y fraterna, de tal modo que los varones podían vivir las dimensiones laborales, sociales y públicas, en la medida en que las mujeres les aseguraran las condiciones necesarias para ello a través de la gestión de la dependencia en el ámbito privado. La noción de ciudadanía, aplicada a hombres libres e independientes, fue posible porque había un contrato sexual que establecía que las mujeres debían hacerse cargo de la dependencia masculina para que éstos pudieran ser independientes en la esfera pública (Rodríguez 2010; Pateman 1998).

El modelo existente de ciudadanía posee, entonces, características masculinas, en el cual la vida pública se relaciona con la independencia y la dependencia permanece ignorada, atribuyéndosele a las mujeres como algo natural. La gestión de la dependencia se asocia a las mujeres, siendo privatizada y a la vez minusvalorada. Se requiere, pues, la elaboración de un modelo de ciudadanía que sustituya al modelo existente. Se necesita un modelo de ciudadanía que supere la dicotomía entre independencia y dependencia, que asuma más bien una interdependencia entre los seres humanos, como un elemento que define nuestra autonomía.

En el modelo de cuidadanía, el modelo marshalliano de un ciudadano trabajador que se construye sobre el concepto de dependencia, es reemplazado por un modelo de ciudadano interdependiente, cuidador y receptor de cuidados. El modelo de ciudadanía es, en consecuencia, un modelo masculino, siendo prioritaria la creación de un modelo distinto de ciudadanía que sea capaz de incluir tanto a hombres y mujeres. Este nuevo modelo tiene un espíritu más inclusivo, que puede captarse con la expresión de cuidadanía (Rodríguez 2010).

\section{Análisis de la organización social del cuidado en Bolivia y Chile desde la cuidadanía}

Se postula en este texto que el Estado no es solamente un actor más dentro de la provisión 
de cuidados, sino que es el agente principal en tanto posee un imperativo ético en torno a garantizar a todas las personas las condiciones para relacionarse autónomamente. De acuerdo al enfoque cuidadano, el Estado debe propender a que la interdependencia entre las diversas personas sea reconocida, estableciendo para ello una serie de normas, declaraciones y arreglos institucionales que garanticen la participación de hombres y mujeres en la sociedad en igualdad de oportunidades. A continuación se revisará la organización social del cuidado en Bolivia y Chile, considerando los arreglos que el Estado ha realizado en materia de cuidado infantil, lo que contribuye a superar la visión dicotómica entre personas dependientes e independientes, reconociendo la interdependencia de los seres humanos.

Tanto en Bolivia como en Chile el cuidado infantil ha adquirido especial relevancia en la última década, como lo prueban los avances legislativos, la creación de programas sociales, la consagración de ciertos principios relativos al cuidado en el Plan de lgualdad de Oportunidades $y$, en el caso de Bolivia, su tematización en la Constitución Política del Estado ${ }^{3}$. De esta forma, ambos países manifiestan adelantos en el involucramiento del padre en el periodo posnatal, así como en proporcionar condiciones necesarias, aunque no suficientes, para que la madre pueda reincorporarse al mercado laboral en el periodo posnatal, pre-escolar y escolar. Al facilitar las condiciones para que la mujer logre

El Estado Plurinacional de Bolivia ha asistido en la última década a un periodo de polémicas y ambiciosas reformas políticas a nivel institucional y legal, cuya máxima expresión es la nueva Constitución Política del Estado. Diversos sectores sociales, incluyendo agrupaciones de mujeres, participaron en la Asamblea Constituyente, la que en el año 2007 definió un texto que al año siguiente fue pactado en el Congreso Nacional y aprobado finalmente en referendo popular en 2009 (Wanderley 2011). mantenerse o incorporarse al trabajo, el Estado propende a una cuidadanía, contribuyendo así a que las mujeres puedan liberarse de aquellas relaciones que limitan su autonomía.

En Bolivia, la Ley General de Trabajo otorga a las madres licencia por maternidad e inamovilidad laboral. Así, se establece un periodo de pre y posnatal de 30 días, respectivamente. Además, dispone que las trabajadoras con hijos menores de un año gozarán de inamovilidad laboral. Durante el periodo de lactancia tendrán derecho a un descanso al día de una hora de extensión. Esta ley no faculta a los padres a una licencia por parentalidad, aunque el Decreto Supremo № 0012 en el año 2009 extendió la inamovilidad laboral a los padres con hijos menores de un año, con independencia incluso de su estado civil. Existe, asimismo, una reglamentación sobre fomento a la lactancia materna ${ }^{4}$, la cual dispone que los organismos públicos y privados deben permitir a las madres llevar a sus bebés a su trabajo y a su estudio en periodo de lactancia, durante los seis primeros meses de vida.

En el caso chileno, las madres gozan de un periodo prenatal de seis semanas y un periodo posnatal de hasta seis meses, el que fue ampliado desde tres a seis meses el año 2011 mediante la ley 20.545. Una novedad de esta ley es que permite trasladar al padre parte de este permiso, de tal manera que a partir de las segundas doce semanas se pueden transferir seis semanas al padre. Otra medida que involucra al padre es el derecho de paternidad irrenunciable, consistente en cinco días pagados al momento de nacer o adoptar a un hijo (Arriagada y Todaro 2012).

Decreto Supremo 0115 que reglamenta la Ley 3460 sobre fomento a la lactancia materna. 
Se aprecia que el Estado ha incluido en cierta medida al padre en el nacimiento del hijo: en el caso de Chile, con el permiso de cinco días de parentalidad irrenunciable al nacer el bebé, además del posnatal optativo que el padre puede tomar si la madre le cede parte del suyo; en el caso de Bolivia, considerando la inamovilidad laboral de un año luego del nacimiento. La inclusión del padre en el nacimiento de su hijo representa un paso para la instauración de una cuidadanía.

De acuerdo a este modelo, la persona se concibe no como un ser aislado, sino relacional. Se centra en el individuo como una persona integrada a redes relacionales, y reconoce que la formación de la identidad del individuo se define de acuerdo a estas relaciones. Desde la perspectiva relacional, el ser humano se concibe como un ser autónomo, y la autonomía se refiere a nuestra capacidad de autodefinirnos. La ciudadanía social debe ser ejercida entonces no de manera individual, sino relacional. Ejercer la ciudadanía social en términos relacionales implica que el Estado debe garantizar las condiciones para que ciudadanos y ciudadanas puedan relacionarse autónomamente. El Estado debe asumir la obligación de ayudar a las personas a desprenderse de aquellas relaciones que entorpezcan su autonomía, así como favorecer las relaciones que mejor ayudan a definir la identidad de las personas (Rodríguez 2010). A través de las licencias parentales, incluyendo a ambos progenitores, el Estado contribuye a garantizar las condiciones para dichas relaciones de autonomía.

A fin de que la mujer no se vea impedida de trabajar luego de haber tenido hijos, en ambos países la ley garantiza salas cuna, aun cuando el marco institucional debe ser bastante mejorado. Así, en Bolivia, la Ley General de Trabajo establece la obligatoriedad de salas cuna en empresas que tengan más de 50 trabajadores, aunque esta disposición en general no es cumplida ni por instituciones públicas ni privadas (Wanderley 2011).

En el caso de Chile, el Código del Trabajo dispone que las empresas deben poseer una sala cuna cuando haya 20 y más mujeres trabajando en la firma. Esto resulta insuficiente y además produce discriminación. Atendiendo a ello, el Plan de Igualdad de Oportunidades 20112020 establece entre sus metas: "Reformar el Código del Trabajo de modo de eliminar las discriminaciones en contra de la contratación de la mujer, modificando el artículo 203 que establece sólo para el empleador que contrata más de 20 trabajadores en una empresa la obligación de pagar una sala cuna" (SERNAM 2011: 45).

En el último decenio ha habido avances en las políticas públicas de ambos países orientados a la salud de los infantes. En Bolivia, para atender la salud de la madre y del bebé se estableció el bono Juana Azurduy de Padilla, que busca prevenir las causas de la mortalidad materna en las familias más pobres, incentivando a las mujeres embarazadas a asistir a controles médicos en el periodo de gestación. El bono proporciona un pago de 50 bolivianos por cada uno de los primeros cuatro controles prenatales, más 120 bolivianos como ayuda para el parto. Dispone igualmente un pago de 125 bolivianos cada vez que las madres acudan con sus niños a consultas médicas bimensuales, hasta que el niño cumpla los dos años (Wanderley 2011).

En Chile, durante el primer gobierno de Bachelet (2006-2010), se implementó el programa Chile 
Crece Contigo, que apunta al cuidado de niños y niñas. Comienza con el primer control de embarazo de la madre en el consultorio de salud respectivo, hasta la incorporación del infante al sistema escolar a los cuatro años de edad (MIDEPLAN 2010). Este programa se concibe como un sistema de protección integral a la infancia y entrega prestaciones fundamentalmente de salud y educativas, destacándose la creación de salas cuna gratuitas para niños y niñas menores de dos años de edad, además de un subsidio familiar prenatal. El programa está orientado al $40 \%$ de la población más pobre y a las madres que trabajan, buscan trabajo, estudian o presentan una situación de vulnerabilidad (Arriagada y Todaro 2012).

En ambos países se han implementado sistemas de atención pre-escolar y escolar, lo que incide positivamente en la participación laboral femenina. En Bolivia se han implementado centros o servicios de atención infantil para la población pre-escolar (de 0 a 4 años), y centros de atención complementarios a la escuela para escolares (de 5 a 18 años) (Farah et al. 2012). Además, se dispuso un bono -bono Juancito Pinto- que incentiva la permanencia escolar, orientado a niños y niñas que asisten a escuelas públicas cursando entre primero y quinto de primaria. El bono consiste en un monto anual de 200 bolivianos y cubre a más de un millón de infantes, estimándose una cobertura del 97\% de los alumnos en condición de pobreza (Wanderley 2011).

Por su parte, el programa Chile Crece Contigo extendió la asistencia para niños de 2 a 4 años de edad a los jardines infantiles, focalizado en los grupos socioeconómicos de menores ingresos o en condición de vulnerabilidad. Otro programa orientado al cuidado de niños en
Chile es el Programa de 4 a 7, ofrecido por el Servicio Nacional de la Mujer (SERNAM), el cual tiene por objetivo posibilitar la inserción laboral de las madres o mujeres responsables del cuidado de niños entre 6 y 13 años, a través de apoyo educativo y recreativo una vez finalizada la jornada escolar. Se les ofrece a niños y niñas un programa de talleres educativos que los apoyen en sus deberes escolares $\mathrm{y}$, además, actividades recreativas, deportivas, sociales, artísticas y culturales que brinden una formación integral.

Las medidas implementadas por los Estados para una atención pre-escolar y escolar de los infantes, así como atenciones orientadas a la salud, contribuyen a una mayor autonomía de las madres. De esta manera, las mujeres pueden tener la opción de generar ingresos participando en el mercadolaboral, contribuyendoal bienestar económico de su familia. La cuidadanía entiende la dependencia y la independencia como un elemento constitutivo de nuestra autonomía. Dependemos de nuestras relaciones para formar nuestra personalidad, y somos también independientes de nuestras relaciones en la medida que podemos emitir un juicio crítico de ellas y alejarnos de aquellas que entorpecen nuestra autonomía. El reconocimiento de esta dependencia e independencia implica revalorizar las tareas de cuidado (Rodríguez 2010). El apoyo del Estado es, entonces, necesario para superar la visión en términos dicotómicos de dependencia e independencia, y comprenderla como interdependencia.

Además de los programas, bonos y modificaciones legales para el cuidado de niñas y niños, se establecen en ambos gobiernos declaraciones programáticas consagradas en el Plan de Igualdad de Oportunidades. En 
Bolivia se dictó el Plan Nacional para la Igualdad de Oportunidades en el año 2008, el que incorpora el tema del cuidado y de las políticas de conciliación entre la vida familiar y laboral. Sobre el ámbito laboral se menciona la necesidad de visibilización del trabajo doméstico y su contabilización en las estadísticas nacionales. Se asume, asimismo, que la continuidad del trabajo de las mujeres en el mercado laboral es afectada por sus responsabilidades familiares (Wanderley 2011).

Con el objetivo de mejorar la autonomía económica de las mujeres, el Plan Nacional para la Igualdad de Oportunidades recomienda dentro de las políticas "redistribuir las tareas de cuidado y protección de la familia entre mujeres $y$ hombres dentro del hogar y entre las familias y el Estado" (Viceministerio de Género y Asuntos Generacionales 2008: 104). Resulta interesante notar que el Plan Nacional para la Igualdad de Oportunidades reconoce de manera implícita tanto la corresponsabilidad entre hombres y mujeres, como la corresponsabilidad del Estado en materia de cuidado.

En el caso de Chile, el Plan de Igualdad de Oportunidades 2011-2020 considera la corresponsabilidad y el cuidado. Se consagra el principio de la corresponsabilidad social, referido tanto a la corresponsabilidad entre hombres y mujeres, así como a la corresponsabilidad ente la familia y el Estado en materia de cuidado de personas dependientes. En el plano familiar, la corresponsabilidad se entiende como una "distribución de tareas que permita compartir de manera más equitativa las obligaciones de los padres en el cuidado y mantención de la casa, los hijos y los adultos mayores incorporados al núcleo familiar, considerando la nueva realidad laboral y social de la mujer" (SERNAM 2011:
25). En el plano estatal, "nos habla de dar pasos firmes hacia una cultura respetuosa de las diferencias entre hombres y mujeres, pero también respetuosa de la igualdad de derechos y oportunidades que ambos merecen, y que esto se refleje en la legislación y en las prácticas y productos culturales" (SERNAM 2011: 25).

Según declara el programa, el Estado se hace cargo de una política de igualdad de oportunidades que considera la participación de los varones en las tareas de cuidado:

\begin{abstract}
Esto debe hacerse favoreciendo que los hombres participen en plenitud en la crianza de sus hijos e hijas, compartan equitativamente las tareas de cuidado, además de las otras tareas propias del hogar, disfruten de los afectos y la paternidad en el mundo privado y en su desarrollo personal, y que aspiren a ser valorados no sólo por su capacidad de proveer y mandar, sino por su capacidad de educar a las nuevas generaciones (SERNAM 2011: 35).
\end{abstract}

En el documento se consagra, además, la necesidad de que tanto el mundo privado como el público generen las transformaciones institucionales que se requieren para dar cumplimiento a la corresponsabilidad social. Sobre el cuidado de niños se establece como meta para el decenio 2011-2020:

Fomentar que la educación escolar y los medios
de comunicación y culturales promuevan modelos
de maternidad, paternidad y masculinidad, así
como formas de convivencia familiar y social más
participativos que contribuyan a una cultura de
corresponsabilidad. Haciendo compatible el trabajo
con la familia de modo que hombres y mujeres
tengan horarios flexibles, permisos compartidos,
sistema de apoyo al cuidado infantil, etc. (SERNAM
2011: 36).

En todo el orbe se ha comenzado a visibilizar el cuidado de personas dependientes y cómo esta 
tarea colabora a un bienestar, reconociendo, además, el aporte económico que realizan las mujeres a las economías nacionales (EspingAndersen 1999). En los países aquí analizados la labor de cuidado que normalmente realizan las mujeres y el mayor involucramiento que el Estado y los varones deben tener en esta materia está siendo objeto de declaraciones programáticas, de acuerdo a lo expuesto en los Planes de Igualdad de Oportunidades. En ambos países se reconoce el principio de la corresponsabilidad en el cuidado de menores; por un lado, corresponsabilidad entre mujeres y varones, y por otro, corresponsabilidad entre la familia y el Estado.

Las declaraciones contenidas en los planes, así como el principio de corresponsabilidad en su doble vertiente, reflejan que se está transitando lentamente desde una ciudadanía hacia una cuidadanía. Instaurar un modelo de cuidadanía implica repensar el Estado bajo el prisma de la sociedad civil. El reconocimiento de la naturaleza relacional de las personas en el centro de la ciudadanía desdibuja la separación entre las esferas pública y privada. Si antes la independencia se asociaba con el mundo público y el mundo privado era el espacio donde se vivía la dependencia, al reconocer la interdependencia entre los seres humanos la dimensión de la dependencia de la persona entra a la esfera pública, conformando la definición de ciudadanía política como un ámbito en el cual cuidado y dependencia también tienen su lugar (Rodríguez 2010).

Un ejemplo de este tránsito a una cuidadanía es la Constitución Política de Bolivia, la que otorga especial relevancia al cuidado infantil. Así, la Constitución garantiza la inamovilidad de las mujeres que se encuentren embarazadas, hasta que el infante cumpla un año de edad. Se establece además que las mujeres tienen derecho a una maternidad segura y que el Estado les brindará especial asistencia y protección social tanto durante el embarazo como en el parto y en el periodo posnatal. Además, la Constitución boliviana considera que el trabajo doméstico debe ser visibilizado y reconocido (Wanderley 2011), estableciendo en su artículo 338 que: "el Estado reconocerá el valor económico del trabajo del hogar como fuente de riqueza y deberá cuantificarse en las cuentas públicas". Fue también reconocida la equidad que debe existir en las responsabilidades de hombres y mujeres sobre el cuidado de sus niños, sobre lo cual afirma el artículo 64 que: "Los cónyuges o convivientes tienen el deber de atender, en igualdad de condiciones y mediante el esfuerzo común, el mantenimiento y responsabilidad del hogar, la educación y formación integral de las hijas e hijos mientras sean menores o tengan alguna discapacidad" 5 .

Otro ejemplo de tránsito a la cuidadanía lo constituye la Política Municipal de cuidado que se ha implementado en los últimos años en la ciudad de La Paz. Esta política ha estado a cargo del CIDES-UMSA (Centro de Investigación del Desarrollo, Estudios de Posgrado, dependientes de la Universidad Mayor de San Andrés) y de Conexión -Fondo de Emancipación-, la que se lleva a cabo bajo la modalidad de investigación-acción (Farah et al. 2012).

\footnotetext{
Así como la Nueva Constitución presenta aciertos en materia de cuidado, se observan importantes omisiones. Una de ellas es que no incluyó el cuidado como un derecho que el Estado debe garantizar, y tampoco está mencionado el principio de corresponsabilidad entre el Estado, el mercado, la familia y la comunidad (Wanderley 2011).
} 


\section{Conclusiones}

El cuidado de personas dependientes constituye una necesidad que todas las sociedades deben satisfacer. Las labores de cuidado han sido realizadas tradicionalmente por mujeres, aun cuando existen otros actores que también se han ocupado de ellas. De esta forma, la organización social del cuidado (Daly y Lewis 2000; Lewis 1998) analiza la provisión de cuidado que la familia, el mercado, la comunidad organizada y el Estado proporcionan a la sociedad. En el presente texto se examina la participación estatal en las prestaciones de cuidado, optando por un análisis macro de la organización social del cuidado. El estudio se restringe a la contribución realizada por el Estado al cuidado de infantes, para lo cual se han revisado políticas públicas así como diversas leyes que contribuyen al cuidado de infantes en Bolivia y en Chile, analizándolas desde la perspectiva teórica de la cuidadanía.

El análisis de la participación estatal en la organización social del cuidado en ambos países cobra relevancia por el aumento de la migración boliviana hacia Chile en el último quinquenio, que según el Departamento de Extranjería y Migración aumentó desde un $6,8 \%$ a un $7,7 \%$, contabilizándose al año 201434.201 ciudadanos bolivianos con un permiso de residencia definitivo. Las migrantes bolivianas que Ilegan a Chile, debido a su baja calificación, se desempeñan normalmente en labores domésticas y de cuidado (Carrasco 1998), dejando a sus hijos en Bolivia (Tapia y Ramos 2013; Ramos y Urbina 2012), con lo cual se conforman cadenas globales de cuidado entre ambos países. En este contexto, se ha analizado aquí la contribución del Estado a la provisión de cuidado infantil en Bolivia como país de origen y en Chile como país de destino.
La participación estatal en las tareas de cuidado contribuye a un cambio del modelo de ciudadanía marshalliano, el que supone un hombre independiente en la esfera pública, presentándose como correlato una dependencia en la vida privada. Así, una vida pública independiente es posible porque existe una vida privada con las necesidades de dependencia cubiertas. Por el contrario, el enfoque de la cuidadanía postula que la división entre personas dependientes e independientes no es tal, sino que existe una interdependencia entre los seres humanos (Bodelón 2010).

Los arreglos institucionales realizados tanto por Bolivia como por Chile en materias de cuidado permiten dar pasos hacia una cuidadanía, entendiéndola como un modelo de ciudadanía más inclusivo, en el que las tareas de cuidado son reconocidas. Se ha puesto de relieve que ambos países han realizado en la última década importantes avances sobre el cuidado de infantes. Así, se han dictado leyes específicas que contribuyen al cuidado de niños: en Bolivia, por ejemplo, la ley de inamovilidad laboral del padre y de la madre hasta un año luego de nacido el bebé; en Chile, en tanto, el permiso posnatal de seis meses permite una cierta transferencia de este tiempo al padre. Se han dictado programas públicos: en Bolivia, el Bono Juana Azurduy de Padilla, que incentiva los controles médicos desde la gestación del bebé hasta los dos años de vida; en Chile, el programa Chile Crece Contigo, que aumentó la atención a infantes en salas cuna, y el Programa de 4 a 7, que posibilita dejar a los niños en una institución estatal determinadas horas para que la madre pueda trabajar.

Además, en ambos países se consagra en el Plan de Igualdad de Oportunidades el rol de la sociedad en el cuidado de sus niños, 
fomentándose no sólo una corresponsabilidad entre hombres y mujeres, sino también entre el Estado y las familias. Desde el punto de vista de la cuidadanía, ambos Estados han ido realizando transformaciones institucionales para garantizar la autonomía de todas las personas. Al exhibir el Estado una participación activa en la organización social del cuidado, está promoviendo la cuidadanía como modelo de ciudadanía que considera la interdependencia entre los individuos y, con ello, las necesidades de cuidado de la población. Los arreglos institucionales que tanto Bolivia como Chile han realizado para cubrir prestaciones de cuidado están favoreciendo las relaciones que permiten nuestra autonomía, liberándonos de aquellas que la entorpecen.

Una diferencia central en ambos países sobre la responsabilidad de hombres y mujeres en el cuidado de sus niños está dada por el papel que juega la Constitución Política de cada país ${ }^{6}$. La carta fundamental chilena manifiesta una total ausencia de equidad de varones y mujeres en el cuidado de sus hijos, tornándose en un documento anquilosado que no se condice con los tiempos actuales ni en términos de derechos sustantivos ni en términos de un rol del Estado más activo en materia de corresponsabilidad. Por el contrario, en la Constitución de Bolivia, dictada por el gobierno de Evo Morales hace unos años, se establece que tanto hombres y mujeres, sean éstos cónyuges o convivientes, tienen el deber de atender en igualdad de condiciones a sus niños ${ }^{7}$.

Mientras en Bolivia el gobierno de Evo Morales dictó una nueva carta magna, a través de una Asamblea Constituyente que entró en vigencia en el año 2009, en Chile aún rige la Constitución que dictara Pinochet en 1981.

Aun cuando pueda ser discutible el grado efectivo de involucramiento en las tareas de cuidado que los padres bolivianos presentan, se destaca en este análisis el reconocimiento del deber de cuidado en la carta fundamental.
Por otra parte, si bien en ambos países el Estado debe actualizar sus marcos institucionales en lo relativo a la organización social del cuidado, éstos deben realizarse con distintos énfasis: la nueva Constitución Política del Estado boliviano, más progresista que la chilena en cuanto a corresponsabilidad y cuidado de infantes, abre la necesidad de adecuación del marco legal vigente. Por el contrario, la promulgación en Chile de leyes y la creación de programas públicos que favorecen el cuidado de niños requieren una adecuación de la Constitución Política a esta nueva visión de la sociedad que considera en primer plano la corresponsabilidad familiar y estatal. Para avanzar aún más hacia una cuidadanía es menester, por tanto, que el Estado muestre una coherencia entre los programas y normas concretas que apuntan a las tareas de cuidado, y las declaraciones programáticas y cartas fundamentales que consagran la corresponsabilidad. De no hacerlo, el enfoque cuidadano corre el riesgo de quedar a medio camino, con la consecuente limitación de autonomía que significaría para las mujeres.

Además de los aspectos señalados, tanto los niveles de cobertura como la focalización en las personas de más bajos recursos son aspectos que deben ser mejorados. Según Molano, Robert y García (2012), en América Latina la escasa participación del Estado en materia de cuidados está focalizada, por lo general, en hogares pobres, y está por ello muy alejada de poder cubrir realmente las demandas de cuidado del conjunto de la población. En este punto resulta crucial reconocer que el Estado tiene un papel fundamental en la garantización de los derechos. La cuidadanía entiende a las personas no como seres individuales, sino de manera relacional, lo que tiene su correlato en 
una concepción relacional de sus derechos. Así, nuestros derechos ya no son vistos en función de proteger nuestra individualidad, sino de ayudarnos a mantener las relaciones que posibilitan nuestra autonomía y a liberarnos de las relaciones que la entorpecen (Rodríguez 2010). Por tanto, a través de la creación de leyes que contribuyan a apoyar las labores de cuidado para toda la población que lo requiera, los Estados propenderán a una consolidación del modelo cuidadano.

Por último, para que la cuidadanía pueda efectivamente ser ejercida por hombres $y$ mujeres deben romperse las falsas dicotomías de esfera pública y privada, personal y social. Deben visibilizarse las diversidades, posturas y necesidades, a fin de que puedan ser satisfechas de una manera real por la sociedad (Rodríguez 2010). Mientras tanto, advertimos cómo lentamente Bolivia y Chile van dando tímidos pasos hacia una corresponsabilidad familiar y estatal. Porque a pesar de que los países latinoamericanos estudiados han ido avanzando en su participación estatal dentro de la organización social del cuidado de infantes, aún quedan bastantes arreglos institucionales por realizar.

\section{Bibliografía}

Acosta, E. 2015. Cuidados en crisis. Mujeres migrantes hacia España y Chile. Deusto: Deusto Publicaciones. 2013a. "Mujeres migrantes cuidadoras en flujos migratorios sur-sur y sur-norte: Expectativas, experiencias y valoraciones". Polis 12 (35): 35-62.

2013b. "Entre la necesidad y el no reconocimiento: La valoración de la dimensión temporal en las estrategias familiares para la contratación de cuidadoras domésticas inmigrantes en España y Chile". Si Somos Americanos 13 (2): 141-164.

2011. "Valorar los cuidados al estudiar las migraciones: La crisis del trabajo de cuidado y la feminización de la inmigración en Chile". Mujeres inmigrantes en Chile: ¿Mano de obra o trabajadoras con derechos? Stefoni, C. (Ed.). Santiago: Ediciones Universidad Alberto Hurtado. 195-228.

Aguirre, R. 2007. "Los cuidados familiares como problema público y objeto de políticas". Familias $y$ políticas públicas en América Latina. Una historia de desencuentros. Arriagada, I. (Coord.). Santiago: CEPAL/ UNFPA. 291-300.

, García, C. y Carrasco, C. 2005. El tiempo, los tiempos, una vara de desigualdad. Santiago: CEPAL.

Anderson, J. 2012. La migración femenina peruana en las cadenas globales de cuidados en Chile y España: Transferencia de cuidados y desigualdades de género. Lima: ONU Mujeres.

Ariza, M. 2012. "Vida familiar transnacional en inmigrantes de México y República Dominicana en dos contextos de recepción". Si Somos Americanos 12 (1): 17-47.

Arriagada, I. y Todaro, R. 2012. Cadenas globales de cuidado. El papel de las migrantes peruanas en la provisión de cuidados en Chile. Santo Domingo: INSTRAW-ONU Mujeres.

. y Moreno, M. 2011. "La constitución de cadenas globales de cuidado y las condiciones laborales de las trabajadoras peruanas en Chile". Mujeres inmigrantes en Chile: ¿Mano de obra o trabajadora con derechos? Stefoni, C. (Ed.). Santiago: Ediciones Universidad Alberto Hurtado. 151-191.

Arriaza, P. 2008. Claves de la integración social de los inmigrantes extranjeros: El caso de las inmigrantes peruanas(os) y bolivianas(os) participantes en la Pastoral de Migración de la ciudad de lquique. Su inserción en la ciudad, sus características y sus demandas sociales. lquique: FOSIS.

Batthyány, K., Genta, N. y Perrotta, V. 2013. "El discurso experto sobre el cuidado desde una perspectiva de género: Análisis comparativo entre el cuidado infantil y de adultos/as mayores en Uruguay". Revista Austral de Ciencias Sociales 25: 23-46.

2004. Cuidado infantil y trabajo. ¿Un desafío exclusivamente femenino? Montevideo: CINTERFOR.

Berganza, I. y Cerna, M. 2011. Dinámicas migratorias en la frontera Perú-Chile. Arica, Tacna e Iquique. Lima: 
Fondo Editorial de la Universidad Ruiz de Montoya.

Bettio, F., Simmonazzi, A. y Villa, P. 2006. "Change in care regimes and female migration: "The care drain" in the Mediterranean". Journal of European Social Policy 16 (3): 271-295.

Bodelón, E. 2010. "Leyes de igualdad en Europa y transformaciones de la ciudadanía". Derecho, género e igualdad. Cambios en las estructuras jurídicas androcéntricas. Vol. I. Heim, D. y Bodelón, E. (Coords.). Barcelona: Universidad Autónoma de Barcelona. 9-26.

Carcedo, A., Lexartza, L. y Chaves, M. J. 2011. Cadenas globales de cuidados: El papel de las migrantes nicaragüenses en la provisión de cuidados en Costa Rica. Santo Domingo: ONU Mujeres.

Carrasco, A. M. 1998. "Mujeres aymaras e inserción laboral". Revista de Ciencias Sociales (Cl) 8: 83-96.

Carrasco, C., Borderías, C. y Torns, T. 2011. "Introducción. El trabajo de cuidados: Antecedentes históricos y debates actuales". El trabajo de cuidados: Historia, teoría y políticas. Carrasco, C., Borderías, C. y Torns, T. (Eds.). Madrid: Los Libros de la Catarata. 13-95.

Daly, M. y Lewis, J. 2000. "The concept of social care and the analysis of contemporary welfare states". British Journal of Sociology 51 (2): 281-298.

Departamento de Extranjería y Migración. 2010. Informe anual del Departamento de Extranjería y Migración. Santiago: Ministerio del Interior.

Duncan, S. y Pfau-Effinger, B. 2000. Gender, economy and culture in the European Union. London: Routledge.

Durán, M. Á. 2004. "La conciliación entre vida y empleo". Cuadernos de Información Económica 183: 129134.

Esping-Andersen, G. 2002. "A new gender contract". Why we need a new welfare state. Esping-Andersen, G. et al. (Eds.). New York: Oxford University Press. 68-95.

1999. Social foundations of postindustrial economies. New York: Oxford University Press.

Farah, I. et al. 2012. Hacia una política municipal del cuidado. La Paz: Posgrado en Ciencias del Desarrollo CIDES-UMSA.

Fundación Scalabrini. 2014. Los derechos de las personas migrantes y la gobernanza de las migraciones en Chile. Jornadas Migratorias XVI. En línea, disponible en: http://fundacionscalabrini.cl/wpcontent/uploads/2014/09/JM-20141.pdf_(visitado el 7 de noviembre de 2014).

Gavilán, V. y Tapia, M. 2006. "Diagnóstico de los procesos migratorios en el norte de Chile". Revista Electrónica Parinas 2 (1). En línea, disponible en: http://www.unap.cl/p4_inte/site/artic/20060807/ pags/20060807194044.html (visitado el 7 de noviembre de 2014).

González, H. 1998. Características de la inserción de aymaras chilenos y bolivianos en el área de Arica. Arica: Taller de Estudios Andinos.

Gonzálvez, H. 2013. "Los cuidados en el centro de la migración. La organización social de los cuidados transnacionales desde un enfoque de género". Migraciones 33: 127-153.

Guaygua, G. 2010. La familia transnacional. Cambios en las relaciones sociales y familiares de migrantes de El Alto y La Paz a España. La Paz: CLACSO-Fundación Programa de Investigación Estratégica en Bolivia.

Hinojosa, A. 2010. La visibilización de las migraciones transnacionales en Bolivia. Tinkazos 13 (1): 243-259. 2009. Buscando la vida. Familias bolivianas transnacionales en España. La Paz: CLACSO-Fundación Programa de Investigación Estratégica en Bolivia.

Hochschild, A. R. 2001. "Las cadenas mundiales de afecto y asistencia y la plusvalía emocional". En el límite: La vida en el capitalismo global. Giddens, A. y Hutton, W. (Eds.). Barcelona: Tusquets. 187-208.

Hondagneu-Sotelo, P. y Ávila, E. 1997. "'I'm here, but I'm there': The meanings of Latina transnational motherhood". Gender \& Society 11 (5): 548-571.

Instituto Nacional de Estadísticas. 2014. Bolivia, características de población y vivienda. Censo nacional de población y vivienda 2012. Estado Plurinacional de Bolivia: Instituto Nacional de Estadísticas.

Lewis, J. 1998. Gender, social care, and welfare state restructuring in Europe. Aldershot: Ashgate.

Marshall, T. H. 1963. "Citizenship and social class". Sociology at the crossroad and other essays. Marshall, T. H. (Autor). London: Heineman. 67-127.

Martínez Franzoni, J. y Voorend, K. 2013. "Desigualdades de género en los regímenes de bienestar latinoamericanos: Mercado, política social y organización familiar". Las fronteras del cuidado. Agenda, derechos e infraestructura. Pautassi, L. y Zibecchi, C. (Coords.). Buenos Aires: Equipo Latinoamericano de Justicia y Género-Editorial Biblos. 59-97.

Martínez, J. 2003. El encanto de los datos. Sociodemografía de la inmigración en Chile según el censo de 2002. Santiago: CEPAL.

MIDEPLAN. 2012. Situación ocupacional, previsional e ingresos del trabajo. Encuesta CASEN 2011. Santiago: Ministerio de Planificación.

2010. Cuatro años creciendo juntos. Memoria del sistema de protección integral a la infancia Chile Crece Contigo 2006-2010. Santiago: Gráfica Puerto Madero. 
Molano, A., Robert, E. y García, M. 2012. Cadenas globales de cuidados: Síntesis de resultados de nueve estudios en América Latina y España. Santo Domingo: INSTRAW-ONU Mujeres.

Montaño, S. et al. 2012. Matriz de análisis para la identificación de políticas justas de igualdad de género. Santiago: CEPAL.

2010. "El cuidado en acción". El cuidado en acción. Entre el derecho y el trabajo. Montaño, S. y Calderón, C. (Coords.). Santiago: CEPAL-AECID-UNIFEM. 13-65.

OIM. 2011. Perfil migratorio de Bolivia. Buenos Aires: Organización Internacional para las Migraciones.

Parella, S. 2003. Mujer, inmigrante y trabajadora. La triple discriminación. Barcelona: Anthropos.

Pateman, C. 1988. The Sexual Contract. Stanford: Stanford University Press.

Pautassi, L. 2013. "Perspectivas actuales en torno al enfoque de derechos y cuidado: La autonomía en tensión". Las fronteras del cuidado. Agenda, derechos e infraestructura. Pautassi, L. y Zibecchi, C. (Coords.). Buenos Aires: Equipo Latinoamericano de Justicia y Género-Editorial Biblos. 99-132.

2010. "Cuidado y derechos: La nueva cuestión social". Entre el derecho y el trabajo. Montaño, S. y Calderón, C. (Coords.). Santiago: CEPAL-AECID-UNIFEM. 69-92.

Pérez, A. y López, S. 2011. Desigualdades a flor de piel: Cadenas globales de cuidados. Concreciones en el empleo de hogar y articulaciones políticas. Navarra: INSTRAW-ONU Mujeres.

2009. Miradas globales a la organización social de los cuidados en tiempos de crisis l: ¿Qué está ocurriendo? Santo Domingo: INSTRAW-ONU Mujeres. 2007. Cadenas globales de cuidado. Santo Domingo: INSTRAW-ONU Mujeres.

2006. Perspectivas feministas en torno a la economía: El caso de los cuidados. Madrid: CES.

Pfau-Effinger, B. 2014. "New policies for caring family members in European welfare states". Cuadernos de Relaciones Laborales 32 (1): 33-48.

Ramos, R. y Urbina, D. 2012. Percepción de las mujeres inmigrantes usuarias de la Pastoral de Migraciones IncamiIquique en cuanto a su vinculación con las redes de apoyo en la región de Tarapacá durante el segundo semestre del año 2011. Tesis para optar al grado académico de Licenciado en Trabajo Social. Iquique: Universidad Arturo Prat.

Rodríguez, B. 2010. "Hacia un estado post-patriarcal. Feminismo y ciudadanía". Revista de Estudios Políticos 149: 87-122.
Sanchís, N. y Rodríguez, C. 2011. Cadenas globales de cuidado. El papel de las migrantes paraguayas en la provisión de cuidados en Argentina. Buenos Aires: ONU Mujeres.

Salazar, R. 2001. Servants of globalization: Women, migration and domestic work. Stanford: Stanford University Press.

Salazar, C., Jiménez, E. y Wanderley, F. 2010. Migración, cuidado y sostenibilidad de la vida. La Paz: Posgrado en Ciencias del Desarrollo CIDES UMSA.

SERNAM. 2011. Plan de igualdad de oportunidades entre hombres y mujeres 2011-2020. Hacia un Chile justo y corresponsable. Santiago: Servicio Nacional de la Mujer.

Setién, M. L. y Acosta, E. 2011. "Cuidados y flujos migratorios feminizados sur-norte y sur-sur: Negación de derechos y ciudadanía limitada". Revista Latina de Sociología 1: 182-208.

Stefoni, C. 2013. "Los cibercafé como lugares de prácticas trasnacionales: El caso de la maternidad a distancia". Polis 12 (35). En línea, disponible en: http:// polis.revues.org/9362 (visitado el 7 de noviembre de 2014).

2009. "Migración, género y servicio doméstico. Mujeres peruanas en Chile". Trabajo doméstico: Un largo camino hacia el trabajo decente. Valenzuela, M. E. y Mora, C. (Eds.). Santiago: Oficina Internacional del Trabajo. 191232.

Tapia, M. y Ramos, R. 2013. "Mujeres migrantes fronterizas en Tarapacá a principios del siglo XXI. El cruce de las fronteras y las redes de apoyo". Polis 12 (35). En línea, disponible en: http://polis.revues.org/9321 visitado el 7 de noviembre de 2014).

2012. "Frontera y migración en el Norte de Chile a partir del análisis de los censos de población. Siglos XIX-XXI". Revista de Geografía Norte Grande 53: 177-198. 2010a. "Inmigración boliviana en España. Un caso para la comprensión de la migración internacional con perspectiva de género". Tinkazos 13 (28): 109-127.

2010b. Yo venía con un sueño... Relaciones de género entre inmigrantes de origen boliviano en Madrid. 2000-2007. Madrid: Universidad Complutense-Instituto de Investigación José Ortega y Gasset.

Torns, T. 2008. "El trabajo y el cuidado: Cuestiones teórico metodológicas desde la perspectiva de género". EMPIRIA. Revista de Metodología de Ciencias Sociales 15: 53-73.

Vergara, M. 2005. El trabajo de los extranjeros en Chile y en la I Región. Santiago: Departamento de Estudios de la Dirección del Trabajo.

Viceministerio de Género y Asuntos Generacionales. 
2008. Plan nacional para la igualdad de oportunidades. Mujeres construyendo la nueva Bolivia para vivir bien. La Paz: Viceministerio de Género y Asuntos Generacionales.
Wanderley, F. 2011. El cuidado como derecho social: Situación y desafíos del bienestar social en Bolivia. Santiago: Oficina Internacional del Trabajo. 
